Entropy 2006, 8[2], 44-49

Entropy

ISSN 1099-4300

www.mdpi.org/entropy/

letter to the Editor

\title{
Remarks on the Compatibility of Opposite Arrows of Time II
}

H. D. Zeh

Universität Heidelberg

postal address: Gaiberger Str. 38, D69151 Waldhilsbach, Germany

www.zeh-hd.de

E-mail: zeh@urz.uni-heidelberg.de

Received: 13 March 2006 / Accepted: 4 April 2006 / Published: 5 April 2006

Abstract: In a series of papers [1, 2, 3], Lawrence Schulman presented examples which demonstrate the compatibility of opposite arrows of time in various situations. In a previous letter to this journal [4] I questioned some of them for not being realistic in spite of being logically correct. Schulman replied [5] to these objections in a letter directly succeeding my one. I am here trying to clarify some aspects of the dispute, thereby further explaining and supporting my previous conclusions.

Keywords: time arrow, final conditions, solvable models, causality, retardation, quantum measurements, gravitational entropy, cosmology. 


\section{Introduction}

It is always an intellectual pleasure arguing with Larry Schulman, in particular on fundamental problems such as the arrow of time. Let me therefore first emphasize that this discussion can be meaningful (and hopefully useful for the reader) only because we already agree on many basic assumptions - for example, that an arrow of time is not intrinsic to the concept of time (as traditionally assumed by philosophers), but the consequence of boundary conditions which seem to characterize this specific universe. Although even this may ultimately be a matter of definition (of time), the boundary value approach is the appropriate one in a setting that is based on physical laws which are deterministic and, up to certain "compensating symmetry transformations" [6], even symmetric under time reversal. The physical meaning of the determinism of the Schrödinger equation is, of course, itself a fundamental issue (see Sect. 4).

For example, we seem to agree that the global arrow of time could as well point in the opposite formal direction (although this would not make an observable difference), that there might be no arrow at all, or that there could be opposite arrows in dynamically isolated systems (such as causally disconnected regions of spacetime). We also seem to agree that the arrow of time characterizing most physical and physiological processes may in principle change direction if and when the universe starts recontracting, while the nature of phenomena occurring in the transition region (which may have to last quite a while) would then probably have to be quite unusual, and presumably exclude anything resembling memory or information.

So our remaining problem may be described as whether or not this transition can be drastically inhomogeneous, such that local "pockets" of an arrow may persist beyond the turning point of cosmic expansion, and remain consistent with the then globally dominating opposite arrow. Since two non-interacting universes with opposite arrows could trivially co-exist, the answer to this problem must depend on the strength of the interaction between the pockets and the rest - and here we still disagree. For example, it would be particularly fascinating to observe from outside "time going backward" in such a pocket. However, we are no Laplacean demons with their unrestricted observation capacities and independent arrows, but participators of this universe. Since sufficiently isolated pockets can hardly be expected to exist here on earth or within the solar system, it appears technically difficult, for example, to "illuminate them with our own light" for this purpose. While their light would be advanced from our point of view, we should nonetheless be able to see it, since in a classical description there is but one (the "real") electromagnetic field that interacts with our retina - although the latter would then react in our causal direction.

Even if we could use our own light for this purpose, it would slightly disturb matter in the pocket. So the question arises, what this would mean in a situation with varying direction of "causality". This problem is illustrated in Fig. 2 of [4] by means of the exact microscopic evolution of a system consisting of many degrees of freedom. This evolution is assumed to start from a state of low entropy and, for statistical reasons, then to evolve into a high-entropy state. If a very small change of the microscopic state is now assumed at a certain time, one may re-calculate the trajectory from this new boundary condition in both directions of time. While the evolution would in general only microscopically be changed in what used to be the direction of growing 
entropy (the physical future), it becomes macroscopically different in the opposite direction - as demonstrated in the figure by means of the entropy. Hence, a small change of state in the "time pocket" must be expected to drastically change its history (defined as its own past - that is, in the direction of our future), thereby destroying its previously assumed opposite arrow. ${ }^{1}$ Even if we assume that this distortion, caused by us, induced changes in the pocket only in its future (in the direction of our past), it would then also somewhere have to affect our past, and, by the same argument, destroy the low entropy condition at the big bang that our existence relies on.

This consequence, which seems to demonstrate the inconsistence of arbitrary boundary conditions at different times, is a probable result, obtained for a "typical" (arbitrarily chosen) solution taken from the pocket. However, Schulman's argument is more subtle. His question is whether solutions which obey boundary conditions at both ends exist (even though they may be very improbable). Indeed, for the same reason that any global solution which characterizes an arrow is improbable when compared with the overwhelming majority of quasi-equilibrium states, but nonetheless readily accepted as describing reality, we should similarly be ready to accept even less probable solutions which obey two low entropy boundary conditions if there are reasons to consider such conditions as given. One may even speculate whether an appropriate assumption of this kind could give rise to a unique solution for cosmic evolution - given the kinematical concepts and dynamical laws. Since anti-causality is counterintuitive, one cannot refer to causal intuition in order to disprove its possibility.

As we discussed various consequences of this situation in our previous letters and former publications, I will now briefly address some of Schulman's arguments in his reply to my objections, essentially in the order he presented them.

\section{Wet carpets and detective stories}

In his wet carpet example [2], Schulman explained how a warning from the future by means of retarded radiation to close the window and save one's carpet from getting wet by a sudden rain shower may be consistent with determinism. Similarly, Wheeler and Feynman [7] had discussed the case of a bullet passing a trap door and causing this door to close before the passage by means of advanced radiation sent from the space behind the door. Would the door or the window close because of the warning, or not, since there would be no warning if it was closed? In both cases, a consistent solution of the problem requires a continuum of possible states in between a closed and an open door, that is, the possibility of a half-open door. This continuum is related to the infinite information capacity of classical phase space, that will be quantitatively discussed and compared with the existence of phase space cells in the next section.

However, while these examples are correct and helpful for an understanding, they are also unrealistic in neglecting uncontrollable but nonetheless important degrees of freedom, which are responsible for the "overdetermination of the past" that characterizes a causal world and lets the past appear fixed. In quantum theory, this unavoidable information spreading leads to the

\footnotetext{
${ }^{1}$ It would be illustrative to generalize this model to a set of local degrees of freedom with local interactions in order to study the propagation of the distortion in space, and the resulting "causal structures".
} 
phenomenon of decoherence. For this reason I compared these examples, which employ isolated systems, with certain clever science fiction stories that describe time travel into the past in an apparently consistent manner - namely by neglecting all uncontrollable phenomena which are related to irreversiblity. A simliar strategy is known for good detective stories, where the murderer must come from a given set of persons (in contrast to crime in reality), and is expected to be "logically determined" by only those events which were explicitly mentioned in the story.

Of course, I did not want to say that Schulman's or Wheeler and Feynman's important examples represent science fiction. Quite the contrary: they are very informative, even though they neglect an important aspect that must be added in order to describe the real world.

\section{Phase space volume}

In order to comply with quantum theory, an effective classical phase space must be assumed to consist of finite cells of size $h^{3 N}$, where $3 N$ is the number of degrees of freedom. In my arguments I had raised the question if a two-times boundary condition of low entropy would possibly reduce phase space to less than one cell - thus signalling dynamical inconsistence in the case that the conditions can be regarded as statistically independent. Schulman replied that a typical phase space volume for a gas would contain something like $10^{10^{20.28}}$ phase space cells. A reduction of the spatial volume by a factor of 64 , say, would reduce the second exponent only in the second figure after the decimal point, such that this reduction could easily be applied twice.

This is correct, but arguments with double exponentials may easily be deceiving: a small entropy difference may require a huge probability ratio. For simplicity, let me consider a gas consisting of $N=10^{20}$ particles. Under customary conditions here on earth, the phase space for each particle in a gas is of the order $10^{10} h^{3}$. The resulting $N$-particle phase space $\left(10^{10}\right)^{N} h^{3 N}=10^{10^{21}} h^{3 N}$ is slightly larger than Schulman's choice. Reducing the single-particle phase space by a factor of 64 would indeed lead to a very "small" change, given by $\left(10^{10} / 64\right)^{N}=10^{10^{20.916}}$, although it repesents a reduction of phase space by a factor of $(1 / 64)^{10^{20}}$. Even forgetting Gibbs' paradox and enlarging phase space by the enormous factor of $N ! \approx N^{N}$ would lead to "no more" than $\left(10^{20} 10^{10}\right)^{N}=10^{10^{21.477}}$. The entropy is therefore almost exclusively determined by the particle number $N$.

On the other hand, entropy differences in the solar system are governed by density and temperature ratios such as those between the sun and interstellar space. So, a change of single-particle phase space by a factor of $10^{5}$ (that is, a reduction from $10^{10}$ to $10^{5}$ ) in an irreversible cosmic process appears quite conservative, and would be far from requiring degenerate matter. Applied once, it reduces total phase space to $\left(10^{5}\right)^{N}=10^{10^{20.699}}$ - apparently not drastically different from the numbers given above, but applied twice (at two sufficiently distant times) it leads to $\left(10^{0}\right)^{N}=1$ (independent of $N)$ ! A similar consistency problem would arise in Wheeler and Feynman's timesymmetric absorber theory (see Chap. 2 of $[6]$ ).

In cosmological context, entropy is dominated by gravity and black holes (see Sect. 5). A twotimes low entropy condition would then lead to severe consistency problems even in the case of a simultaneous transition between opposite arrows. They may perhaps be overcome in time-less 
quantum gravity [8].

\section{Quantum measurement}

Schulman claims furthermore that "special quantum states" (obeying appropriate final conditions) would be able to solve the quantum measurement problem without requiring deviations from unitary dynamics. This would be a dramatic achievement, but it could not be explained just by assuming separate "pockets" with an opposite arrow. It would instead require special final conditions whenever and wherever measurements or measurement-like events occur. Special initial conditions of low entropy are in general readily accepted, since their causal origin can be confirmed "historically" by means of consistent documents. No such documents exist about a low entropy future. Simply assuming "special states" in this way, just as they are required, would therefore replace science by arbitrary wishful thinking.

However, there exist arguments which seem to strictly rule out such possibilities by investigating the dynamics of individual quantum measurements [9]. They are based on von Neumann's interaction, and valid regardless of all individual complexity in the device, such as the presence of many particles or metastable states. No freedom for a selection of final states then remains except for the addition of irrelevant degrees of freedom.

In fact, the example defined in Schulmann's section 6.2 of [1] is not concerned with a superposition of different measurement results, but with a superposition of just two states: one with a droplet somewhere in the Wilson chamber (itself a superposition of many locations), and one with no droplet at all (no measurement). Moreover, the system is treated as closed, hence neglecting any decoherence, which would necessarily characterize a visible droplet or any other "pointer position". The model, which uses a spin lattice, leads to a final state that oscillates in time between "droplet" (here represented by many correlated spin flips) and "no droplet", and so allows one to assume ad hoc that the coupling between these two states happens to cease at "special times", namely when one of the two oscillating amplitudes vanishes. However, such oscillations are known to occur only for isolated "pathological" systems, such as harmononic oscillators, while realistic complex systems behave irreversibly. As mentioned above, this model would not solve the problem of superpositions of different pointer positions, while the superposition considered by Schulman could easily be avoided by an irreversible formation of droplets (with $100 \%$ efficiency rather than oscillation). Superpositions of different pointer positions would in fact immediately and irreversibly be decohered by the environment, and thereafter be dynamically robust (with coefficients remaining essentially fixed), but continue to exist in different Everett worlds if the Schrödinger equation were universally valid. Schulman's proposal to solve the measurement problem does not even seem to be possible as 'wishful thinking'.

\section{Gravity}

It has been known at least since Bekenstein's discovery of black hole entropy and Penrose's subsequent estimates [10] that cosmic entropy is overwhelmingly dominated by gravity. The major 
low entropy property of the universe is its homogenity. The formation of inhomogeneities in the form of stars, galaxies and ultimately black holes defines the most important process of entropy production - regardless of what happens inside the event horizon of a black hole, or what kind of new physics may apply there or close to the horizon [11]. It is often overlooked that solar (or even larger) black holes would lose mass by radiation only in the very distant future of an ever expanding universe: at present their accumulation of $3 K$ background radiation by far outways their $10^{-7} \mathrm{~K}$ (or weaker) Hawking radiation. In order to get rid of gravitating objects for a cosmic "time reversal" in the not extremely distant future, one would need advanced (incoming coherent) radiation to reverse their gravitational contraction [8]. These objects are, therefore, strongly coupled to the general arrow of time, and a further indication that the arrow cannot vary from place to place.

To conclude, let me emphasize again that situations with opposite arrows may be very useful for pedagogical purposes, but can not be expected to "really happen". Therefore, they appear particularly misleading when presented as possibilities to solve the quantum measurement problem.

\section{References}

[1] Schulman, L.S., "Time's arrows and quantum mechanics" (Cambridge University Press, 1997).

[2] Schulman, L.S., Phys. Rev. Lett. 83, 5419 (1999).

[3] Schulman, L.S. , in: "Time's Arrow, Quantum Measurements and Superluminal Behavior", Edts. D. Mugnai, A. Ranfagni and L.S. Schulman (Consiglio Nazionale delle Ricerche, Roma 2001) - cond-mat/0102071.

[4] Zeh, H.D., Entropy 7[4], 199 (2005) - http://www.mdpi.org/entropy/list05.htm\#issue4.

[5] Schulman, L.S., Entropy 7[4], 208 (2005) - http://www.mdpi.org/entropy/list05.htm\#issue4.

[6] Zeh, H.D., "The Physical Basis of the Direction of Time" (Springer, Berlin 2001).

[7] Wheeler, J.A., and Feynman, R.P., Rev. Mod. Phys. 21, 425 (1949).

[8] Kiefer, C., and Zeh, H.D., Phys. Rev. D51, 4145 (1995).

[9] Wigner, E.P., Am. J. Phys. 31, 6 (1963); Zeh, H.D., Found. Phys. 1, 69 (1970) - both reprinted in Wheeler, J.A., and Zurek W.H., "Quantum Theory and Measurement" (Princeton UP 1983).

[10] Penrose, R., in: "Quantum Gravity II", Edts. Isham, C.J., Penrose. R., and Sciama, D.W. (Clarendon Press, 1981).

[11] Zeh, H.D., Phys. Lett. A347, 1, (2005).

(c)2006 by MDPI (http://www.mdpi.org). Reproduction for noncommercial purposes permitted. 